Article

\title{
Synthesis of [(4-Chloro-5H-1,2,3-dithiazol-5-ylidene)amino]azines
}

\author{
Panayiotis A. Koutentis *, Maria Koyioni and Sophia S. Michaelidou \\ Department of Chemistry, University of Cyprus, P.O. Box 20537, 1678 Nicosia, Cyprus; \\ E-Mails: maria_koyioni@hotmail.com (M.K.), sophie_michaelidou@hotmail.com (S.S.M.) \\ * Author to whom correspondence should be addressed; E-Mail: koutenti@ucy.ac.cy; \\ Tel.: +357-22-892783; Fax: +357-22-892809.
}

Received: 26 September 2011; in revised form: 21 October 2011 / Accepted: 21 October 2011 / Published: 25 October 2011

\begin{abstract}
The reactions of 2-, 3- and 4-aminopyridines with 4,5-dichloro-1,2,3-dithiazolium chloride (Appel salt) 4 to give $N$-(4-chloro-5H-1,2,3-dithiazol-5-ylidene)pyridin-Xamines 1a $(X=2), 1 \mathbf{g}(X=3)$ and $1 \mathbf{k}(X=4)$ were optimized with respect to base, temperature and reaction time. Based on these conditions a total of thirteen [(dithiazolylidene)amino]azines 1a-m were prepared and fully characterized.
\end{abstract}

Keywords: heterocycle; Appel salt; 1,2,3-dithiazoles; condensation; azine

\section{Introduction}

Select $\quad N$-(4-chloro-5H-1,2,3-dithiazol-5-ylidene)anilines $\quad(N$-aryl-1,2,3-dithiazol-5 $H$-imines $) \quad$ show interesting antitumor [1], antibacterial [2-4], antifungal [5-7], and herbicidal [8] activity. The biological activity could be due to the 1,2,3-dithiazole ring, that acts as an inhibitor of several enzymes that are structurally related to serine proteases [9]. Furthermore, $N$-aryldithiazolimines are useful precursors to other heterocycles. For example the thermolysis of $N$-aryldithiazolimines can afford benzothiazoles [10,11], benzimidazoles [12], thiazolopyridines [13] and benzoxazines [14]. Moreover, $N$-aryldithiazolimines can also be transformed into useful acyclic functionalities such as cyanothio-formanilides [15-17], $N$-arylcyanoformimidoyl chlorides $[10,18]$ and $N$-arylisothiocyanates $[19,20]$.

Despite their synthetic utility the library of dithiazolimines prepared from Appel salt $\mathbf{4}$ is comprised mainly of analogues where the dithiazolimine moiety is bound to a benzene ring. Some examples also exist where the dithiazole moiety is bound to 5-membered heteroles [21-23], but to the best of our 
knowledge the only reported examples, where the dithiazolimine moiety is bound directly to a 6-membered azine, are $\mathrm{N}$-(4-chloro-5H-1,2,3-dithiazol-5-ylidene)pyridin-2-amine (1a) [24], $N^{3}$-(4-chloro-5H-1,2,3-dithiazol-5-ylidene)- $N^{2}$-phenylpyridine-2,3-diamine (2) [25] and $N^{2}, N^{3}$-bis(4chloro-5H-1,2,3-dithiazol-5-ylidene)pyridine-2,3-diamine (3) [25] (Figure 1).

Figure 1. Structures of known $\mathrm{N}$-(4-chloro-5H-1,2,3-dithiazol-5-ylidene)pyridinamines.

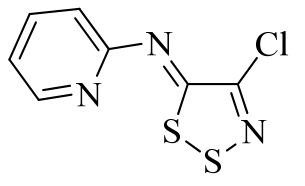

$1 \mathbf{a}$

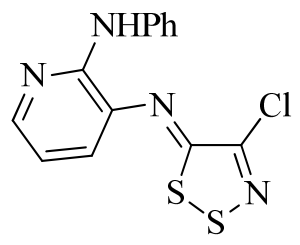

2

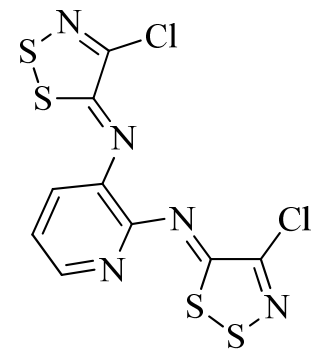

3

This may be due to the fact that electron poor aminoazines, such as aminopyridines, have less nucleophilic character than primary anilines. This can lead to low yields of the desired [(4-chloro-5H1,2,3-dithiazolylidene)amino]azines and/or complex reaction mixtures due to side reactions [26]. As such, it was decided to study the synthesis of the less explored [(4-chloro-5H-1,2,3-dithiazolylidene)amino]azines. Herein we wish to report our results.

\section{Results and Discussion}

\subsection{Studies on N-(4-Chloro-5H-1,2,3-dithiazol-5-ylidene)pyridinamines}

Our investigation began with the reaction of the simplest aminoazines, namely aminopyridines, with Appel salt 4 to afford $N$-(4-chloro-5H-1,2,3-dithiazolylidene)pyridin-X-amines (where $\mathrm{X}=2,3$ and 4). By focusing of the 2-, 3- and 4-aminopyridines we hoped to see how the position of the pyridyl ring nitrogen affected the reactivity of the aminoazine with Appel salt 4. These reactions were optimized with respect to base, temperature, and reaction time (Scheme 1).

Scheme 1. Preparation of $N$-(4-chloro-5H-1,2,3-dithiazol-5-ylidene)pyridinamines 1a, $1 \mathbf{g}$ and $\mathbf{1 k}$.

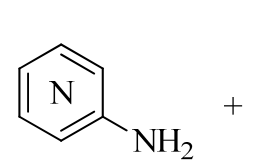

pyrid-2-yl

pyrid-3-yl

pyrid-4-yl $\underset{\text { ii) } \operatorname{Py}(2 \text { equiv. }), D C M, 20{ }^{\circ} \mathrm{C}, 2 \mathrm{~h}}{\longrightarrow}$<smiles>[Hg]S[Hg]</smiles>

4<smiles>Clc1nss/c1=N\c1ccccc1</smiles>

1a pyrid-2-yl $(69 \%)$

1 pyrid-3-yl (24\%)

$1 \mathrm{k}$ pyrid-4-yl $(1 \%)$

Initially the synthesis of $N$-(4-chloro-5H-1,2,3-dithiazol-5-ylidene)pyridinamines $\mathbf{1 a}, \mathbf{1 g}$ and $\mathbf{1 k}$ was based on a well established literature procedure for the preparation of (dithiazolylideneamino)arenes [24], using pyridine (2 equiv.) as base, at ca. $20{ }^{\circ} \mathrm{C}$ in DCM (Scheme 1). It became clear even at this stage that the product yields were affected by the position of the pyridyl nitrogen: The reaction between 2-, 
3- and 4-aminopyridines and Appel salt 4 gave the corresponding $\mathrm{N}$-(4-chloro-5H-1,2,3-dithiazol-5ylidene)pyridin-X-amines $\mathbf{1 a}(X=2), \mathbf{1 g}(X=3)$ and $\mathbf{1 k}(X=4)$ in 69,24 and $1 \%$ yields, respectively.

To improve the yields in the above reactions we then screened a variety of amine bases. Weakly aromatic amine bases like pyridine ( $\left.\mathrm{p} K_{\mathrm{b}} 8.8\right)$ and the more sterically demanding (less nucleophilic) lutidine $\left(\mathrm{p} K_{\mathrm{b}}\right.$ 7.4) were included, as well as a range of trialkylamines with increasing steric demands, reduced nucleophilicity and increasing basicity e.g., $\mathrm{DABCO}\left(\mathrm{p} K_{\mathrm{b}} 5.2\right), \mathrm{Et}_{3} \mathrm{~N}\left(\mathrm{p} K_{\mathrm{b}} 3.4\right)$, and Hünig's base ( $\left.\mathrm{p} K_{\mathrm{b}} 2.6\right)$, and "weakly" nucleophilic strong amidine bases such as DBU $\left(\mathrm{p} K_{\mathrm{b}} 1.1\right)$ and $\mathrm{DBN}\left(\mathrm{p} K_{\mathrm{b}} 0.5\right)$ [27].

The addition of base was needed to obtain greater than trace quantities of (dithiazolylidene)pyridinamines. Furthermore, increasing the reaction time (by 4, 6 and $8 \mathrm{~h}$ ) before or after the addition of base, decreased the yields. Increasing the reaction temperature from 25 to $40{ }^{\circ} \mathrm{C}$ also did not lead to an improvement of the observed yields. The best conditions required mixing Appel salt 4 with the aminopyridine for $1 \mathrm{~h}$ at room temperature followed by the addition of amine base (2 equiv.) and a further $2 \mathrm{~h}$ of stirring (Table 1$)$.

Table 1. Reaction of Appel salt $4(2.18 \mathrm{mmol})$ with aminopyridines $(2.18 \mathrm{mmol})$ in DCM $(4 \mathrm{~mL})$ at $c a .20{ }^{\circ} \mathrm{C}$ for $1 \mathrm{~h}$ and followed by base $(4.36 \mathrm{mmol})$ for another $2 \mathrm{~h}$.

\begin{tabular}{|c|c|c|}
\hline Aminopyridine & Base $\left(p K_{b}\right)$ & Yields of $1 \mathrm{a}, 1 \mathrm{~g}$ or $1 \mathrm{k}(\%)$ \\
\hline \multirow{7}{*}{ Pyridin-2-amine } & Pyridine (8.8) & 1a $(69)$ \\
\hline & 2,6-Lutidine (7.4) & 1a $(72)$ \\
\hline & Dabco (5.2) & 1a $(55)$ \\
\hline & $\mathrm{Et}_{3} \mathrm{~N}(3.4)$ & 1a $(69)$ \\
\hline & i- $\operatorname{Pr}_{2} \operatorname{NEt}(2.6)$ & 1a $(73)$ \\
\hline & DBU (1.1) & 1a $(58)$ \\
\hline & DBN $(0.5)$ & 1a (47) \\
\hline \multirow{7}{*}{ Pyridin-3-amine } & Pyridine (8.8) & $\lg (24)$ \\
\hline & 2,6-Lutidine (7.4) & $\lg (45)$ \\
\hline & Dabco (5.2) & $\lg (8)$ \\
\hline & $\mathrm{Et}_{3} \mathrm{~N}(3.4)$ & $1 \mathrm{~g}(43)$ \\
\hline & i- $-\operatorname{Pr}_{2} \operatorname{NEt}(2.6)$ & $\lg (57)$ \\
\hline & DBU (1.1) & $\lg (16)$ \\
\hline & DBN $(0.5)$ & $\lg (13)$ \\
\hline \multirow{7}{*}{ Pyridin-4-amine } & Pyridine (8.8) & $\mathbf{1 k}$ (traces) \\
\hline & 2,6-Lutidine (7.4) & $\mathbf{1 k}$ (traces) \\
\hline & Dabco (5.2) & $1 \mathbf{k}(3)$ \\
\hline & $\mathrm{Et}_{3} \mathrm{~N}(3.4)$ & $1 \mathbf{k}(23)$ \\
\hline & $\mathrm{i}-\mathrm{Pr}_{2} \mathrm{NEt}(2.6)$ & $\mathbf{1 k}(13)$ \\
\hline & DBU (1.1) & $\mathbf{1 k}(13)$ \\
\hline & $\mathrm{DBN}(0.5)$ & $\mathbf{1 k}$ (traces) \\
\hline
\end{tabular}

The highest yields of $N$-(4-chloro-5H-1,2,3-dithiazol-5-ylidene)pyridin-2-amine (1a) and $N$-(4chloro-5H-1,2,3-dithiazol-5-ylidene)pyridin-3-amine (1g), 73 and 57\%, respectively, were obtained with Hünig's base, while the highest yield for $\mathrm{N}$-(4-chloro-5H-1,2,3-dithiazol-5-ylidene)pyridin-4amine (1k) (23\%) was obtained with $\mathrm{Et}_{3} \mathrm{~N}$. The exceptionally low yields of product from the reaction of 4-aminopyridine and Appel salt $\mathbf{4}$ may be partially explained by the reduced nucleophilicity of the 
primary amine, which is due to a significant contribution of a zwitterionic resonance form. This deactivation is not observed with 2-aminopyridine since the contribution of the analogous zwitterionic resonance form is considerably less important (Scheme 2) [28]. Moreover, 4-aminopyridine was not very soluble in the solvents tried (DCM, $\mathrm{PhH}, \mathrm{MeOH}, \mathrm{EtOH}$,) and this could also have contributed to the low product yields.

Scheme 2. Zwitterionic resonance forms for 2- and 4-aminopyridines.<smiles>Nc1ccccn1</smiles><smiles></smiles><smiles>Nc1ccncc1</smiles><smiles>[NH2+]=c1cc[nH]cc1</smiles>

Also notable was that the reaction between 2-aminopyridine and Appel salt $\mathbf{4}$ was less sensitive to the base used, which tentatively may be attributed to two factors: (1) The pyrid-2-yl nitrogen's ability to coordinate with the dithiazole sulfur S-1 in a "non-bonding" manner [29,30] provided particularly stable (dithiazolylidene)pyridinamines; and (2) the acidity of the proton in intermediate $\mathbf{6}$ was enhanced by both the neighbouring pyridyl nitrogen and the positively charged dithiazolium ring sulfur [9,23]. Both these features could lead to a very facile base catalysed elimination of $\mathrm{HCl}$ (Scheme 3 ).

Scheme 3. Proposed intermediates for the synthesis of (dithiazolylidene)pyridin-2-amine 7.

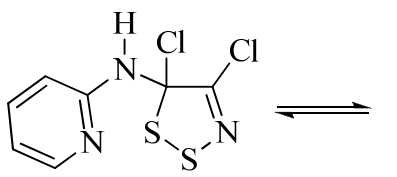

5<smiles>Clc1ns[s+]c1Nc1ccccn1</smiles>

$\mathrm{Cl}^{-}$

6

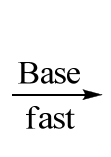<smiles>Clc1nss/c1=N\c1ccccn1</smiles><smiles>Clc1ns[s+]c1N=c1cccc[n-]1</smiles>

\subsection{Synthesis of a [(4-Chloro-5H-1,2,3-dithiazol-5-ylidene)amino] azine Library}

To investigate this further, a range of substituted aminopyridines and related azines were reacted with Appel salt 4 in the presence of the above amine bases (Table 2). In nearly all cases the substituents ( $\mathrm{Me}, \mathrm{Hal}, \mathrm{CN}$, and $\mathrm{NO}_{2}$ ) on the aminoazine had little effect on the product yields. The exception to this was the use of 3-aminopyridin-2-one, which only gave good yields $(53 \%)$ of the dithiazolylideneamine $\mathbf{1 h}$ when pyridine was used as base. As before, the position of the nitrogen atom in the aromatic ring affected the product yields. In the case of 2-and 3-amino derivatives the desired [(dithiazolylidene)amino]azines were obtained in moderate to good yields. 4-Aminopyridines gave very low yields with all the bases as expected; however, the presence of an additional nitrogen atom $\alpha$ to the amine as in 4-aminopyrimidine-5-carbonitrile led to the formation of the dithiazolylideneamine $\mathbf{1 m}$ in moderate to good yields (15-61\%). 
Table 2. Reaction of 4,5-dichloro-1,2,3-dithiazolium chloride 4 (2.18 mmol) with aminoazines $(2.18 \mathrm{mmol})$ in DCM $(4 \mathrm{~mL})$ at $c a .20{ }^{\circ} \mathrm{C}$ for $1 \mathrm{~h}$ and then addition of base $(4.36 \mathrm{mmol})$ for $2 \mathrm{~h}$ to give [(4-chloro-5H-1,2,3-dithiazolylidene)amino]azines $\mathbf{1 a}-\mathbf{m}$.

\begin{tabular}{|c|c|c|c|c|c|c|c|}
\hline \multirow[b]{2}{*}{ Azine } & \multicolumn{7}{|c|}{ Product Yields (\%) } \\
\hline & Pyridine & Lutidine & DABCO & $\mathbf{E t}_{3} \mathbf{N}$ & $\mathrm{i}-\mathrm{Pr}_{2} \mathbf{N E t}$ & DBU & DBN \\
\hline $1 \mathbf{a}($ pyrid-2-yl) & 69 & 72 & 55 & 69 & 73 & 58 & 47 \\
\hline $1 \mathbf{b}(3-\mathrm{MeO}-$ pyrid-2-yl) & 71 & 67 & 55 & 60 & 66 & 42 & 40 \\
\hline 1c $\left(3,5-\mathrm{Cl}_{2}\right.$-pyrid-2-yl $)$ & 69 & 70 & 52 & 44 & 43 & 44 & 39 \\
\hline $\mathbf{1 d}\left(3,5-\mathrm{Br}_{2}\right.$-pyrid-2-yl) & 65 & 70 & 56 & 48 & 40 & 27 & 15 \\
\hline 1 e $\left(3-\mathrm{O}_{2} \mathrm{~N}\right.$-pyrid-2-yl) & 45 & 62 & 48 & 32 & 14 & 23 & 12 \\
\hline 1f (pyrazin-2-yl) & 65 & 63 & 51 & 63 & 61 & 40 & 36 \\
\hline $1 \mathrm{~g}$ (pyrid-3-yl) & 23 & 45 & 8 & 42 & 57 & 16 & 10 \\
\hline 1h (2-HO-pyrid-3-yl) & 53 & 19 & 11 & 9 & 18 & 12 & 5 \\
\hline 1i (2-Cl-pyrid-3-yl) & 75 & 82 & 85 & 71 & 72 & 50 & 45 \\
\hline 1j (4-Cl-pyrid-3-yl) & 65 & 76 & 60 & 70 & 69 & 21 & 19 \\
\hline 1k (pyrid-4-yl) & trace & trace & 3 & 21 & 13 & 13 & 10 \\
\hline $1 \mathbf{l}\left(2,6-\mathrm{Me}_{2}\right.$-pyrid-4-yl) & 5 & trace & 19 & 22 & 10 & 9 & 5 \\
\hline $1 \mathbf{m}$ (5-NC-pyrimid-4-yl) & 61 & 55 & 35 & 45 & 40 & 39 & 15 \\
\hline
\end{tabular}

\section{Experimental}

\subsection{General}

Reactions were protected from atmospheric moisture by $\mathrm{CaCl}_{2}$ drying tubes. Anhydrous $\mathrm{Na}_{2} \mathrm{SO}_{4}$ was used for drying organic extracts, and all volatiles were removed under reduced pressure. All reaction mixtures and column eluents were monitored by TLC using commercial glass backed thin layer chromatography (TLC) plates (Merck Kieselgel $60 \mathrm{~F}_{254}$ ). The plates were observed under UV light at 254 and $365 \mathrm{~nm}$. The technique of dry flash chromatography was used throughout for all non-TLC scale chromatographic separations using Merck Silica Gel 60 (less than $0.063 \mathrm{~mm}$ ). Melting points were determined using a PolyTherm-A, Wagner \& Munz, Koefler-Hotstage Microscope apparatus. Solvents used for recrystallization are indicated after the melting point. UV spectra were obtained using a Perkin-Elmer Lambda-25 UV/vis spectrophotometer and inflections are identified by the abbreviation 'inf'. IR spectra were recorded on a Shimadzu FTIR-NIR Prestige-21 spectrometer with a Pike Miracle Ge ATR accessory and strong, medium and weak peaks are represented by s, $\mathrm{m}$ and w, respectively. ${ }^{1} \mathrm{H}$ - and ${ }^{13} \mathrm{C}$-NMR spectra were recorded on a Bruker Avance 300 instrument (at 300 and $75 \mathrm{MHz}$, respectively). ${ }^{13} \mathrm{C}$-DEPT NMR was used to identify quaternary and tertiary carbons, which are indicated by (s) and (d) notations, respectively. Deuterated solvents were used for homonuclear lock and the signals are referenced to the deuterated solvent peaks. Low resolution (EI) mass spectra were recorded on a Shimadzu Q2010 GC-MS with direct inlet probe.

\subsection{General Procedure for the Synthesis of [(4-Chloro-5H-1,2,3-dithiazol-5-ylidene)amino] azines}

To a stirred solution of 4,5-dichloro-1,2,3-dithiazolium chloride $(4,454.2 \mathrm{mg}, 2.18 \mathrm{mmol})$ in DCM $(4 \mathrm{~mL})$ at $c a .20{ }^{\circ} \mathrm{C}$ and protected with $\mathrm{CaCl}_{2}$ drying tube, was added the corresponding aminoazine 
(1 equiv., $2.18 \mathrm{mmol}$ ). After $1 \mathrm{~h}$, to the reaction mixture was added, dropwise, the appropriate base (2 equiv., $4.36 \mathrm{mmol}$ ) and the mixture left to stir at $c a .20{ }^{\circ} \mathrm{C}$ for additional $2 \mathrm{~h}$. The reaction mixture was subsequently adsorbed onto silica and chromatographed to afford the corresponding [(4-chloro-5H-1,2,3-dithiazol-5-ylidene)amino]azine $\mathbf{1}$.

(Z)-N-(4-Chloro-5H-1,2,3-dithiazol-5-ylidene)pyridin-2-amine (1a): Yellow-green needles (365.6 mg, 73\%), mp 149-150 ${ }^{\circ} \mathrm{C}$ (lit. [24], 154-155 ${ }^{\circ} \mathrm{C}$ ) (cyclohexane); Anal. found: C, 36.6; H, 1.7; N, 18.3. $\mathrm{C}_{7} \mathrm{H}_{4} \mathrm{ClN}_{3} \mathrm{~S}_{2}$ requires $\left.\mathrm{C}, 36.6 ; \mathrm{H}, 1.8 ; \mathrm{N}, 18.3 \%\right) ; \mathrm{UV} \lambda_{\max }(\mathrm{DCM}) / \mathrm{nm} 246$ (log $\varepsilon$ 2.83), 294 (2.48), 388 (2.84), 405 (2.96), 427 (2.76); IR $v_{\max } / \mathrm{cm}^{-1} 1589 \mathrm{w}, 1560 \mathrm{w}, 1512 \mathrm{~m}, 1491 \mathrm{~m}, 1449 \mathrm{~m}, 1431 \mathrm{~m}, 1296 \mathrm{w}$, $1267 \mathrm{w}, 1258 \mathrm{w}, 1175 \mathrm{~m}, 1142 \mathrm{~m}, 1092 \mathrm{w}, 1042 \mathrm{w}, 999 \mathrm{w}, 891 \mathrm{~m}, 862 \mathrm{~m}, 787 \mathrm{~s}, 742 \mathrm{~m}, 704 \mathrm{~m} ;{ }^{1} \mathrm{H}-\mathrm{NMR}$ $\left(\mathrm{CDCl}_{3}\right) \delta_{\mathrm{H}} 8.60(1 \mathrm{H}, \mathrm{d}, J 4.2, H-3$ or 6$), 7.90(1 \mathrm{H}, \mathrm{ddd}, 7.7,7.7,1.7, H-4$ or 5$), 7.67(1 \mathrm{H}, \mathrm{d}, J 8.1, H-3$ or 6), $7.28\left(1 \mathrm{H}\right.$, ddd, $J$ 7.2, 5.1, 1.0, $H-4$ or 5); ${ }^{13} \mathrm{C}-\mathrm{NMR}\left(\mathrm{CDCl}_{3}\right) \delta_{\mathrm{C}} 157.9$ (s), 153.9 (s), 148.9 (s), 143.3 (d), 138.4 (d), 122.4 (d), 121.6 (d); MS m/z (EI) $231\left(\mathrm{M}^{+}+2,8 \%\right), 229\left(\mathrm{M}^{+}, 19\right), 194$ (85), 168 (5), 162 (5), 125 (3), 104 (3), 78 (100), 70 (6), 64 (20), 51 (43).

(Z)-N-(4-Chloro-5H-1,2,3-dithiazol-5-ylidene)-3-methoxypyridin-2-amine (1b): Yellow fibers (402 mg, 71\%), mp 190-191 ${ }^{\circ} \mathrm{C}$ (cyclohexane/EtOH); Anal. found: C, 36.9; H, 2.1; N, 16.3. $\mathrm{C}_{8} \mathrm{H}_{6} \mathrm{ClN}_{3} \mathrm{OS}_{2}$ requires $\mathrm{C}, 37.0 ; \mathrm{H}, 2.3 ; \mathrm{N}, 16.2 \%)$; UV $\lambda_{\max }(\mathrm{DCM}) 228$ (log $\left.\varepsilon 3.69\right), 249$ (3.70), 304 (3.42), $361 \mathrm{inf}$ (3.05), $374 \inf$ (3.33), $386 \inf$ (3.53), 403 (3.78), 425 (3.85), 449 (3.61); IR $v_{\max } / \mathrm{cm}^{-1} 3075 \mathrm{w}, 2970 \mathrm{w}$, $2936 \mathrm{w}, 2837 \mathrm{w}, 1572 \mathrm{~m}, 1508 \mathrm{~m}, 1487 \mathrm{~m}, 1464 \mathrm{~m}, 1449 \mathrm{w}, 1431 \mathrm{~s}, 1425 \mathrm{~s}, 1308 \mathrm{~s}, 1294 \mathrm{~m}, 1279 \mathrm{~s}, 1263 \mathrm{w}$, $1209 \mathrm{w}, 1182 \mathrm{~m}, 1171 \mathrm{~m}, 1155 \mathrm{w}, 1125 \mathrm{~s}, 1076 \mathrm{w}, 1013 \mathrm{~s}, 951 \mathrm{w}, 893 \mathrm{~m}, 868 \mathrm{~s}, 808 \mathrm{~s}, 783 \mathrm{~s}, 773 \mathrm{w}, 764 \mathrm{~s}$; ${ }^{1} \mathrm{H}-\mathrm{NMR}\left(\mathrm{DMSO}-d_{6}\right) \delta_{\mathrm{H}} 8.22(1 \mathrm{H}, \mathrm{d}, J 4.2, H-4$ or 6$), 7.63(1 \mathrm{H}, \mathrm{d}, J 7.8, H-4$ or 6$), 7.43(1 \mathrm{H}, \mathrm{dd}, J 8.1$, 4.8, H-5), $3.95\left(3 \mathrm{H}, \mathrm{s}, \mathrm{CH}_{3} \mathrm{O}\right) ;{ }^{13} \mathrm{C}-\mathrm{NMR}$ (DMSO-d $\left.{ }_{6}\right) \delta_{\mathrm{C}} 156.9(\mathrm{~s}), 150.6(\mathrm{~s}), 148.3$ (s), 144.3 (s), 134.3 (d), 123.3 (d), 119.8 (d), $55.9\left(\mathrm{CH}_{3} \mathrm{O}\right)$; $\mathrm{MS} \mathrm{m} / z$ (EI); $261\left(\mathrm{M}^{+}+2,8 \%\right), 259\left(\mathrm{M}^{+}, 19\right), 226(8)$, 224 (37), 195 (5), 160 (36), 134 (7), 123 (8), 108 (13), 93 (14), 78 (100), 70 (12), 64 (30), 51 (20).

(Z)-3,5-Dichloro-N-(4-chloro-5H-1,2,3-dithiazol-5-ylidene)pyridin-2-amine (1c): Yellow cotton fibers (456 mg, 70\%), mp 145-146 ${ }^{\circ} \mathrm{C}$ (from cyclohexane); Anal. found: C, 28.2; H, 0.6; N, 13.9. $\mathrm{C}_{7} \mathrm{H}_{2} \mathrm{Cl}_{3} \mathrm{~N}_{3} \mathrm{~S}_{2}$ requires $\left.\mathrm{C}, 28.2 ; \mathrm{H}, 0.7 ; \mathrm{N}, 14.1 \%\right) ; \mathrm{UV} \lambda_{\max }(\mathrm{DCM}) 229(\log \varepsilon$ 3.17), 254 (3.30), 261 (3.26), 313 (2.86), $338 \inf (3.07), 397$ (3.31), 418 (3.42), 440 (3.22); IR $v_{\max } / \mathrm{cm}^{-1} 3067 \mathrm{w}, 3049 \mathrm{w}$ (Ar CH), 1564m, 1539m, 1524m, 1491m, 1412s, 1377m, 1279m, 1240m, 1225w, 1171m, 1142w, $1121 \mathrm{~m}, 1061 \mathrm{~m}, 924 \mathrm{w}, 901 \mathrm{~m}, 880 \mathrm{~s}, 810 \mathrm{~m}, 764 \mathrm{~m}, 756 \mathrm{~m} ;{ }^{1} \mathrm{H}-\mathrm{NMR}$ (DMSO-d $\left.{ }_{6}\right) \delta_{\mathrm{H}} 8.64(1 \mathrm{H}, \mathrm{d}, J 2.2$, $H-4$ or 6$), 8.37(1 \mathrm{H}, \mathrm{d}, J 2.2, H-4$ or 6$) ;{ }^{13} \mathrm{C}-\mathrm{NMR}\left(\mathrm{DMSO}-d_{6}\right) \delta_{\mathrm{C}} 160.3(\mathrm{~s}), 150.1$ (s), $148.6(\mathrm{~s})$, 141.6 (d), 138.4 (d), 127.6 (s), 127.5 (s); MS m/z (EI); $301\left(\mathrm{M}^{+}+4,3 \%\right) 299\left(\mathrm{M}^{+}+2,6\right), 297\left(\mathrm{M}^{+}, 6\right)$, 266 (4), 264 (18), 262 (24), 236 (3), 172 (3), 146 (10), 137 (4), 125 (4), 110 (29), 102 (5), 98 (5), $93(8), 85(8), 75(15), 70(16), 64$ (100), 50 (5).

(Z)-3,5-Dibromo-N-(4-chloro-5H-1,2,3-dithiazol-5-ylidene)pyridin-2-amine (1d): Yellow cubes (591 mg, 70\%), mp 174-175 ${ }^{\circ} \mathrm{C}$ (from cyclohexane); Anal. found: $\mathrm{C}, 21.8 ; \mathrm{H}, 0.5 ; \mathrm{N}, 10.8 . \mathrm{C}_{7} \mathrm{H}_{2} \mathrm{Br}_{2} \mathrm{ClN}_{3} \mathrm{~S}_{2}$ requires $\mathrm{C}, 21.7 ; \mathrm{H}, 0.5 ; \mathrm{N}, 10.8 \%)$; UV $\lambda_{\max }(\mathrm{DCM}) 229(\log \varepsilon 3.08), 257$ (3.16), 262 inf (3.11), 316 (2.69), $325 \inf$ (2.59), $365 \inf$ (2.52), 399 (3.16), 419 (3.27), 442 (3.07); UV $v_{\max } / \mathrm{cm}^{-1} 1539 \mathrm{~m}$, $1514 \mathrm{~m}, 1485 \mathrm{~m}, 1454 \mathrm{w}, 1414 \mathrm{~s}, 1364 \mathrm{~m}, 1314 \mathrm{w}, 1275 \mathrm{~m}, 1244 \mathrm{w}, 1231 \mathrm{w}, 1167 \mathrm{~m}, 1123 \mathrm{w}, 1098 \mathrm{~m}$, $1045 \mathrm{~m}, 914 \mathrm{w}, 897 \mathrm{~m}, 891 \mathrm{~m}, 876 \mathrm{~s}, 795 \mathrm{~s}, 754 \mathrm{~m}, 737 \mathrm{~s} ;{ }^{1} \mathrm{H}-\mathrm{NMR}\left(\mathrm{DMSO}-d_{6}\right) \delta_{\mathrm{H}} 8.75(1 \mathrm{H}, \mathrm{s}, H-4$ or 6$)$, 
$8.59(1 \mathrm{H}, \mathrm{s}, H-4$ or 6$) ;{ }^{13} \mathrm{C}-\mathrm{NMR}\left(\mathrm{DMSO}-d_{6}\right) \delta_{\mathrm{C}} 160.3(\mathrm{~s}), 151.1(\mathrm{~s}), 148.6(\mathrm{~s}), 144.3$ (d), 143.9 (d), 118.4 (s), 115.8 (s); MS m/z (EI); $391\left(\mathrm{M}^{+}+6,4 \%\right), 389\left(\mathrm{M}^{+}+4,18\right), 387\left(\mathrm{M}^{+}+2,21\right), 385\left(\mathrm{M}^{+}, 9\right)$, 354 (25), 352 (37), 350 (20), 310 (10), 308 (28), 306 (23), 262 (5), 247 (11), 245 (11), 238 (8), 236 (17), 234 (9), 183 (6), 181 (6), 156 (21), 154 (19), 125 (7), 102 (12), 93 (9), 76 (41), 64 (100), 50 (15).

(Z)-N-(4-Chloro-5H-1,2,3-dithiazol-5-ylidene)-3-nitropyridin-2-amine (1e): Yellow cotton fibers (371 mg, 62\%), mp 188-189 ${ }^{\circ} \mathrm{C}$ (from cyclohexane); Anal. found: C, 30.7; H, 1.0; N, 20.4. $\mathrm{C}_{7} \mathrm{H}_{3} \mathrm{ClN}_{4} \mathrm{O}_{2} \mathrm{~S}_{2}$ requires $\left.\mathrm{C}, 30.6 ; \mathrm{H}, 1.1 ; \mathrm{N}, 20.4 \%\right)$; UV $\lambda_{\max }(\mathrm{DCM}) / \mathrm{nm} 230$ (log $\varepsilon$ 2.93), 278 (2.49), 395 inf (2.90), 411 (3.03), 432 (2.88); IR $v_{\max } / \mathrm{cm}^{-1} 1597 \mathrm{~m}, 1560 \mathrm{~m}, 1526 \mathrm{~s}, 1479 \mathrm{~m}, 1427 \mathrm{~s}, 1362 \mathrm{~m}$, $1344 \mathrm{~s}, 1279 \mathrm{w}, 1261 \mathrm{w}, 1244 \mathrm{w}, 1169 \mathrm{~m}, 1086 \mathrm{w}, 901 \mathrm{~s}, 878 \mathrm{~m}, 847 \mathrm{~s}, 808 \mathrm{~s}, 770 \mathrm{~s}, 706 \mathrm{~m}$; ${ }^{1} \mathrm{H}-\mathrm{NMR}$ $\left(\mathrm{DMSO}-d_{6}\right) \delta_{\mathrm{H}} 8.87(1 \mathrm{H}, \mathrm{dd}, J 5.0,1.5, H-6), 8.53(1 \mathrm{H}, \mathrm{dd}, J 7.9,1.5, H-4), 7.59(1 \mathrm{H}, \mathrm{dd}, J 7.9,5.0$, $H-5) ;{ }^{13} \mathrm{C}-\mathrm{NMR}\left(\mathrm{DMSO}-d_{6}\right) \delta_{\mathrm{C}} 161.9$ (s), 148.6 (s), 148.3 (d), 146.8 (s), 141.6 (s), 133.5 (d), 121.8 (d); MS m/z (EI); $276\left(\mathrm{M}^{+}+2,16 \%\right), 274\left(\mathrm{M}^{+}, 37\right), 239$ (18), 228 (41), 226 (99), 210 (4), 191 (6), 155 (12), 149 (35), 137 (13), 119 (78), 102 (18), 91 (63), 76 (38), 70 (29), 64 (100), 50 (22).

(Z)-N-(4-Chloro-5H-1,2,3-dithiazol-5-ylidene)pyrazin-2-amine (1f): Yellow-orange needles (327 mg, $65 \%$ ), mp 208-209 ${ }^{\circ} \mathrm{C}$ (from cyclohexane/EtOH); Anal. found: C, 31.2; H, 1.2; N, 24.2. $\mathrm{C}_{6} \mathrm{H}_{3} \mathrm{ClN}_{4} \mathrm{~S}_{2}$ requires $\mathrm{C}, 31.2 ; \mathrm{H}, 1.3 ; \mathrm{N}, 24.3 \%)$; UV $\lambda_{\max }(\mathrm{DCM}) 230$ (log $\left.\varepsilon 3.02\right), 243$ (3.02), 252 inf (2.96), 297 (2.72), $322 \inf$ (2.61), $379 \inf$ (2.89), 392 (3.10), 409 (3.23), 430 (3.06); IR $v_{\max } / \mathrm{cm}^{-1} 1526 \mathrm{~s}, 1501 \mathrm{~m}$, $1476 \mathrm{~s}, 1452 \mathrm{~s}, 1406 \mathrm{~s}, 1298 \mathrm{~m}, 1281 \mathrm{~m}, 1179 \mathrm{~s}, 1146 \mathrm{~m}, 1061 \mathrm{~m}, 1016 \mathrm{~m}, 903 \mathrm{~s}, 868 \mathrm{~s}, 845 \mathrm{~s}, 793 \mathrm{~s}, 750 \mathrm{w}$, $714 \mathrm{~m} ;{ }^{1} \mathrm{H}-\mathrm{NMR}\left(\mathrm{DMSO}-d_{6}\right) \delta_{\mathrm{H}} 8.95(1 \mathrm{H}, \mathrm{d}, J 1.2, H-3), 8.72(1 \mathrm{H}, \mathrm{dd}, J 2.7,1.5, H-5), 8.59(1 \mathrm{H}, \mathrm{d}$, $J 2.7, H-6$ ); ${ }^{13} \mathrm{C}-\mathrm{NMR}$ (DMSO- $\left.d_{6}\right) \delta_{\mathrm{C}} 160.6(\mathrm{~s}), 150.6(\mathrm{~s}), 148.5(\mathrm{~s}), 144.7$ (d), $141.0(\mathrm{~d}), 139.3(\mathrm{~d})$; MS m/z (EI) $232\left(\mathrm{M}^{+}+2,14 \%\right), 230\left(\mathrm{M}^{+}, 32\right), 195$ (100), 169 (11), 125 (12), 102 (8), 93 (6), 84 (6), 79 (70), 70 (16), 64 (68), 52 (66).

(Z)-N-(4-Chloro-5H-1,2,3-dithiazol-5-ylidene)pyridin-3-amine (1g): Yellow cotton fibers (285 mg, 57\%), mp $126-127^{\circ} \mathrm{C}$ (from cyclohexane/EtOH); Anal. found: C, 36.7; H, 1.7; N, 18.2. $\mathrm{C}_{7} \mathrm{H}_{4} \mathrm{ClN}_{3} \mathrm{~S}_{2}$ requires $\mathrm{C}, 36.6 ; \mathrm{H}, 1.8 ; \mathrm{N}, 18.3 \%$ ); UV $\lambda_{\max }(\mathrm{DCM}) 231$ ( $\left.\log \varepsilon 2.86\right), 280$ (2.33), 374 (2.65); IR $v_{\max } / \mathrm{cm}^{-1} 3051 \mathrm{w}, 3042 \mathrm{w}, 1570 \mathrm{~s}, 1539 \mathrm{w}, 1504 \mathrm{~m}, 1474 \mathrm{~m}, 1410 \mathrm{~s}, 1327 \mathrm{w}, 1229 \mathrm{~s}, 1192 \mathrm{~m}, 1150 \mathrm{~s}, 1121 \mathrm{w}$, $1099 \mathrm{~m}, 1042 \mathrm{~m}, 1022 \mathrm{~m}, 943 \mathrm{~m}, 914 \mathrm{~m}, 870 \mathrm{~s}, 851 \mathrm{~m}, 812 \mathrm{~m}, 773 \mathrm{~s}, 706 \mathrm{~s} ;{ }^{1} \mathrm{H}-\mathrm{NMR}$ (DMSO- $\left.d_{6}\right) \delta_{\mathrm{H}}$ 8.47-8.43 (2H, m, $H-2$ and 6), 7.65 (1H, ddd, $J$ 8.3, 2.6, 1.5, H-4), 7.51 (1H, dd, $J$ 8.1, 4.8, H-5); ${ }^{13} \mathrm{C}-\mathrm{NMR}\left(\mathrm{DMSO}-d_{6}\right) \delta_{\mathrm{C}} 161.9(\mathrm{~s}), 147.3(\mathrm{~s}), 146.8$ (d), 146.6 (s), 141.5 (d), 126.3 (d), 124.6 (d); MS m/z (EI) $231\left(\mathrm{M}^{+}+2,17 \%\right), 229\left(\mathrm{M}^{+}, 42\right), 168$ (23), 130 (6), 125 (8), 104 (9), 102 (5), 93 (6), 78 (38), 70 (11), $64\left(\mathrm{~S}_{2}, 100\right), 51(47)$.

(Z)-3-(4-Chloro-5H-1,2,3-dithiazol-5-ylideneamino)pyridin-2-ol (1h): Orange dust (284 mg, 53\%), mp 151-152 ${ }^{\circ} \mathrm{C}$ (from pentane/DCM); Anal. found $\mathrm{C}, 34.3 ; \mathrm{H}, 1.7 ; \mathrm{N}, 17.0 . \mathrm{C}_{7} \mathrm{H}_{4} \mathrm{ClN}_{3} \mathrm{OS}_{2}$ requires $\mathrm{C}$, 34.2; H, 1.6; N, 17.1\%); UV $\lambda_{\max }(\mathrm{DCM}) / \mathrm{nm} 228$ (log $\varepsilon$ 2.75), 251 (2.67), 326 (2.64), 347 (2.64), 409 (2.77); IR $v_{\max } / \mathrm{cm}^{-1} 3125 \mathrm{w}(\mathrm{OH}), 2922 \mathrm{w}, 2853 \mathrm{w}, 1638 \mathrm{~s}, 1612 \mathrm{~m}, 1572 \mathrm{~m}, 1547 \mathrm{~m}, 1524 \mathrm{w}, 1472 \mathrm{~m}$, $1433 \mathrm{w}, 1358 \mathrm{w}, 1346 \mathrm{w}, 1323 \mathrm{w}, 1310 \mathrm{w}, 1245 \mathrm{~m}, 1236 \mathrm{~m}, 1175 \mathrm{w}, 1144 \mathrm{~m}, 1057 \mathrm{w}, 1020 \mathrm{w}, 959 \mathrm{w}, 943 \mathrm{~m}$, 910m, 893w, 858s, 826w, 797w, 779w, 770s; ${ }^{1} \mathrm{H}-\mathrm{NMR}\left(\mathrm{DMSO}-d_{6}\right) \delta_{\mathrm{H}} 12.07(1 \mathrm{H}, \mathrm{br}, \mathrm{OH}), 7.37-7.34$ $(2 \mathrm{H}, \mathrm{m}, \mathrm{Py} H), 6.33(1 \mathrm{H}, \mathrm{dd}, J 6.7,6.7$, Py $H) ;{ }^{13} \mathrm{C}-\mathrm{NMR}\left(\mathrm{DMSO}-d_{6}\right) \delta_{\mathrm{C}} 157.5(\mathrm{~s}), 155.1(\mathrm{~s}), 147.0(\mathrm{~s})$, 
137.9 (s), 132.7 (d), 131.2 (d), 105.6 (d); MS m/z (EI) $247\left(\mathrm{M}^{+}+2,9 \%\right), 245\left(\mathrm{M}^{+}, 21\right), 210$ (32), 146 (100), 135 (9), 120 (13), 117 (10), 102 (12), 92 (40), 76 (11), 70 (33), 64 (77), 52 (19).

(Z)-2-Chloro-N-(4-chloro-5H-1,2,3-dithiazol-5-ylidene)pyridin-3-amine (1i): Yellow-orange needles (489.5 mg, 85\%), mp $133-134{ }^{\circ} \mathrm{C}$ (from cyclohexane); Anal. found: C, 31.9; H, 1.0; N, 15.9. $\mathrm{C}_{7} \mathrm{H}_{3} \mathrm{Cl}_{2} \mathrm{~N}_{3} \mathrm{~S}_{2}$ requires $\left.\mathrm{C}, 31.8 ; \mathrm{H}, 1.1 ; \mathrm{N}, 15.9 \%\right) ; \mathrm{UV} \lambda_{\max }(\mathrm{DCM}) 232(\log \varepsilon 2.87), 281$ (2.46), 363 (2.63); IR $v_{\max } / \mathrm{cm}^{-1} 3051 \mathrm{w}, 1722 \mathrm{w}, 1703 \mathrm{w}, 1657 \mathrm{w}, 1584 \mathrm{~s}, 1566 \mathrm{w}, 1557 \mathrm{w}, 1506 \mathrm{~m}, 1443 \mathrm{w}$, $1400 \mathrm{~s}, 1267 \mathrm{w}, 1250 \mathrm{w}, 1240 \mathrm{w}, 1207 \mathrm{~m}, 1157 \mathrm{~m}, 1082 \mathrm{~s}, 1067 \mathrm{w}, 972 \mathrm{w}, 926 \mathrm{w}, 903 \mathrm{w}, 870 \mathrm{~s}, 797 \mathrm{~m}, 779 \mathrm{~s}$, $743 \mathrm{~s}, 710 \mathrm{~s} ;{ }^{1} \mathrm{H}-\mathrm{NMR}\left(\mathrm{CDCl}_{3}\right) \delta_{\mathrm{H}} 8.26(1 \mathrm{H}, \mathrm{d}, J 3.6, H-6), 7.45(1 \mathrm{H}, \mathrm{dd}, J 7.7,1.3, H-4), 7.33(1 \mathrm{H}, \mathrm{dd}$, $J$ 7.8, 4.7, H-5); ${ }^{13} \mathrm{C}-\mathrm{NMR}\left(\mathrm{CDCl}_{3}\right) \delta_{\mathrm{C}} 162.8(\mathrm{~s}), 147.3$ (s), 146.4 (d), $145.0(\mathrm{~s}), 142.5$ (s), 127.3 (d), 123.4 (d); MS m/z (EI); $267\left(\mathrm{M}^{+}+4,4 \%\right), 265\left(\mathrm{M}^{+}+2,21\right), 263\left(\mathrm{M}^{+}, 30\right), 204$ (14), 202 (34), 164 (9), 135 (6), 125 (7), 112 (13), 103 (14), 93 (7), 76 (30), 70 (10), 64 (100), 50 (14).

(Z)-4-Chloro-N-(4-chloro-5H-1,2,3-dithiazol-5-ylidene)pyridin-3-amine (1j): Yellow prisms (437.6 mg, $76 \%$ ), mp $160-161{ }^{\circ} \mathrm{C}$ (from cyclohexane/EtOH); Anal. found: $\mathrm{C}, 31.9 ; \mathrm{H}, 1.1 ; \mathrm{N}, 15.8 . \mathrm{C}_{7} \mathrm{H}_{3} \mathrm{Cl}_{2} \mathrm{~N}_{3} \mathrm{~S}_{2}$ requires $\mathrm{C}, 31.8 ; \mathrm{H}, 1.1 ; \mathrm{N}, 15.9 \%$ ); UV $\lambda_{\max }(\mathrm{DCM}) 231$ ( $\left.\log \varepsilon 2.80\right), 279$ (2.32), 364 (2.59); IR $v_{\max } / \mathrm{cm}^{-1} 3080 \mathrm{w}, 3057 \mathrm{w}, 1591 \mathrm{~s}, 1557 \mathrm{~m}, 1547 \mathrm{w}, 1506 \mathrm{~m}, 1485 \mathrm{w}, 1466 \mathrm{~m}, 1402 \mathrm{~m}, 1283 \mathrm{~m}, 1242 \mathrm{~m}$, $1225 \mathrm{w}, 1215 \mathrm{w}, 1146 \mathrm{~m}, 1092 \mathrm{~s}, 1053 \mathrm{w}, 966 \mathrm{w}, 912 \mathrm{~m}, 862 \mathrm{~s}, 831 \mathrm{~s}, 783 \mathrm{~s}, 739 \mathrm{w}, 708 \mathrm{~s} ;{ }^{1} \mathrm{H}-\mathrm{NMR}$ $\left(\mathrm{DMSO}-d_{6}\right) \delta_{\mathrm{H}} 8.48(1 \mathrm{H}, \mathrm{s}, H-2), 8.40(1 \mathrm{H}, \mathrm{d}, J 5.4, H-5$ or 6$), 7.70(1 \mathrm{H}, \mathrm{d}, J 5.4, H-5$ or 6$) ;{ }^{13} \mathrm{C}-\mathrm{NMR}$ $\left(\mathrm{DMSO}-d_{6}\right) \delta_{\mathrm{C}} 164.5(\mathrm{~s}), 147.4$ (d), 145.7 (s), 145.6 (s), 140.6 (d), 133.4 (s), 125.1 (d); MS $m / z$ (EI) $267\left(\mathrm{M}^{+}+4,3 \%\right), 265\left(\mathrm{M}^{+}+2,20\right), 263\left(\mathrm{M}^{+}, 26\right), 204$ (9), 202 (21), 170 (3), 164 (3), 138 (3), 125 (7), 112 (12), 103 (9), 93 (5), 85 (12), 76 (18), 70 (7), 64 (100), 50 (15).

(Z)-N-(4-Chloro-5H-1,2,3-dithiazol-5-ylidene)pyridin-4-amine (1k): Yellow prisms (120 mg, 24\%), mp 166-167 ${ }^{\circ} \mathrm{C}$ (from cyclohexane/EtOH); Anal. found: $\mathrm{C}, 36.5 ; \mathrm{H}, 1.8 ; \mathrm{N}, 18.1 . \mathrm{C}_{7} \mathrm{H}_{4} \mathrm{ClN}_{3} \mathrm{~S}_{2}$ requires $\mathrm{C}$, 36.6; H, 1.8; N, 18.3\%); UV $\lambda_{\max }(\mathrm{DCM}) 230$ ( $\left.\log \varepsilon 2.82\right), 374$ (2.63); IR $v_{\max } / \mathrm{cm}^{-1} 1593 \mathrm{~m}, 1568 \mathrm{~s}$, $1549 \mathrm{~m}, 1501 \mathrm{~m}, 1485 \mathrm{w}, 1418 \mathrm{~m}, 1246 \mathrm{w}, 1207 \mathrm{~m}, 1152 \mathrm{~m}, 1090 \mathrm{w}, 1053 \mathrm{w}, 999 \mathrm{~m}, 872 \mathrm{~s}, 858 \mathrm{~s}, 827 \mathrm{~m}$, $779 \mathrm{~m}, 735 \mathrm{w} ;{ }^{1} \mathrm{H}-\mathrm{NMR}$ (DMSO- $\left.d_{6}\right) \delta_{\mathrm{H}} 8.63(2 \mathrm{H}, \mathrm{d}, J 5.1, H-2$ and 6$), 7.15(2 \mathrm{H}, \mathrm{dd}, J 4.7,1.4, H-3$ and 5); ${ }^{13} \mathrm{C}-\mathrm{NMR}\left(\mathrm{DMSO}-d_{6}\right) \delta_{\mathrm{C}} 162.5$ (s), 158.1 (s), 151.6 (d), 146.4 (s), 113.9 (d); MS m/z (EI) 231 $\left(\mathrm{M}^{+}+2,34 \%\right), 229\left(\mathrm{M}^{+}, 87\right), 194$ (25), 168 (57), 162 (24), 130 (14), 127 (13), 125 (31), 104 (14), 93 (11), 78 (55), 64 (100), 51 (70).

(Z)-N-(4-Chloro-5H-1,2,3-dithiazol-5-ylidene)-2,6-dimethylpyridin-4-amine (11): Yellow prisms (124 mg, 22\%), mp $127-128{ }^{\circ} \mathrm{C}$ (from cyclohexane/EtOH); Anal. found: C, 42.0; H, 3.1; N, 16.3 . $\mathrm{C}_{9} \mathrm{H}_{8} \mathrm{ClN}_{3} \mathrm{~S}_{2}$ requires $\mathrm{C}, 41.9 ; \mathrm{H}, 3.1 ; \mathrm{N}, 16.3 \%$ ); UV $\lambda_{\max }(\mathrm{DCM}) 331$ (log $\varepsilon 3.01$ ), 248 inf (2.72), $317 \mathrm{inf}(2.28), 370$ (2.75); IR $v_{\max } / \mathrm{cm}^{-1} 2920 \mathrm{w}, 1645 \mathrm{w}, 1584 \mathrm{~s}, 1557 \mathrm{~m}, 1508 \mathrm{~m}, 1454 \mathrm{w}, 1410 \mathrm{w}, 1373 \mathrm{w}$, $1319 \mathrm{~m}, 1292 \mathrm{w}, 1269 \mathrm{w}, 1238 \mathrm{w} .1207 \mathrm{w}, 1171 \mathrm{~s}, 1123 \mathrm{w}, 1024 \mathrm{w}, 995 \mathrm{w}, 939 \mathrm{~m}, 887 \mathrm{~s}, 864 \mathrm{w}, 839 \mathrm{~m}, 779 \mathrm{~s}$, 756w, 733m; ${ }^{1} \mathrm{H}-\mathrm{NMR}\left(\mathrm{CDCl}_{3}\right) \delta_{\mathrm{H}} 6.65(2 \mathrm{H}, \mathrm{s}, \mathrm{H}-2$ and 6$), 2.50\left(6 \mathrm{H}, \mathrm{s}, 2 \times \mathrm{CH}_{3}\right) ;{ }^{13} \mathrm{C}-\mathrm{NMR}\left(\mathrm{CDCl}_{3}\right)$ $\delta_{\mathrm{C}} 160.8(\mathrm{~s}), 160.0(\mathrm{~s}), 159.0(\mathrm{~s}), 147.4(\mathrm{~s}), 109.8(\mathrm{~d}), 24.5\left(\mathrm{CH}_{3}\right) ; \mathrm{MS} \mathrm{m} / z$ (EI) $259\left(\mathrm{M}^{+}+2,36 \%\right), 257$ $\left(\mathrm{M}^{+}, 84\right), 244$ (5), 242 (20), 224 (12), 222 (38), 196 (14), 190 (31), 132 (100), 125 (13), 106 (25), $91(6), 77$ (7), $64(51), 51(6)$. 
(Z)-4-[(4-Chloro-5H-1,2,3-dithiazol-5-ylidene)amino]pyrimidine-5-carbonitrile (1m): Orange prisms (340 mg, 61\%), mp 205-206 ${ }^{\circ} \mathrm{C}$ (from $\mathrm{EtOH}$ ); Anal. found: $\mathrm{C}, 32.9 ; \mathrm{H}, 0.8 ; \mathrm{N}, 27.3 . \mathrm{C}_{7} \mathrm{H}_{2} \mathrm{ClN}_{5} \mathrm{~S}_{2}$ requires $\mathrm{C}, 32.9 ; \mathrm{H}, 0.8 ; \mathrm{N}, 27.4 \%)$; UV $\lambda_{\max }(\mathrm{DCM}) 230$ (log $\left.\varepsilon 2.70\right), 268$ (2.57), $318 \mathrm{inf}$ (2.06), $376 \inf$ (2.50), 395 (2.88), 414 (3.08), 435 (2.99); IR $v_{\max } / \mathrm{cm}^{-1} 2239 \mathrm{w}$ and $2230 \mathrm{w}(\mathrm{C} \equiv \mathrm{N}), 1565 \mathrm{w}$, $1560 \mathrm{w}, 1537 \mathrm{~m}, 1507 \mathrm{~m}, 1459 \mathrm{~s}, 1424 \mathrm{~s}, 1412 \mathrm{~s}, 1391 \mathrm{~s}, 1282 \mathrm{w}, 1192 \mathrm{~m}, 1178 \mathrm{w}, 1162 \mathrm{w}, 1106 \mathrm{w}, 950 \mathrm{w}$, 923s, 876s, 823w, 816m, 787m, 774m; ${ }^{1} \mathrm{H}-\mathrm{NMR}$ (acetone- $\left.d_{6}\right) \delta_{\mathrm{H}} 9.45(1 \mathrm{H}, \mathrm{s}, H-2$ or 6$), 9.28(1 \mathrm{H}, \mathrm{s}$, $H-2$ or 6$) ;{ }^{13} \mathrm{C}-\mathrm{NMR}$ (acetone- $\left.d_{6}\right) \delta_{\mathrm{C}} 166.4(\mathrm{~s}), 163.1(\mathrm{~d}), 162.3(\mathrm{~s}), 159.2(\mathrm{~d}), 150.9(\mathrm{~s}), 114.6(C \equiv \mathrm{N})$, $105.6(C \mathrm{C} \equiv \mathrm{N})$; MS m/z (EI) $257\left(\mathrm{M}^{+}+2,12 \%\right), 255\left(\mathrm{M}^{+}, 27\right), 220$ (87), 194 (9), 125 (10), 104 (11), 102 (8), 93 (8), 77 (55), 70 (12), 64 (100), 51 (17).

\section{Conclusions}

The reaction conditions for the synthesis of a series of [(4-chloro-5H-1,2,3-dithiazolylidene)amino]azines were optimized with respect to base, temperature and reaction time. The optimum conditions involved mixing the aminoazine with Appel salt 4 in DCM at room temperature for $1 \mathrm{~h}$, followed by the addition of amine base ( 2 equiv.) and then an additional $2 \mathrm{~h}$ of stirring at room temperature. Thirteen $N$-heteroazinyl dithiazolimines were successfully synthesized. With 2-pyridylamines, the choice of base was less important than with the 3- and 4-pyridylamines. In these cases the use of trialkylamines such as $\mathrm{Et}_{3} \mathrm{~N}$ and Hünig's base often gave superior product yields.

\section{Acknowledgments}

The authors wish to thank the Cyprus Research Promotion Foundation [Grant Nos.

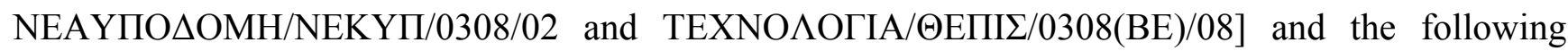
organizations in Cyprus for generous donations of chemicals and glassware: the State General Laboratory, the Agricultural Research Institute, the Ministry of Agriculture and Biotronics Ltd. Furthermore, we thank the A.G. Leventis Foundation for helping to establish the NMR facility in the University of Cyprus.

\section{References}

1. Konstantinova, L.S.; Bol'shakov, O.I.; Obruchnikova, N.V.; Laborie, H.; Tanga, A.; Sopéna, V.; Lanneluc, I.; Picot, L.; Sablé, S.; Thiéry, V.; Rakitin, O.A. One-pot synthesis of 5-phenylimino, 5-thieno or 5-oxo-1,2,3-dithiazoles and evaluation of their antimicrobial and antitumor activity. Bioorg. Med. Chem. Lett. 2009, 19, 136-141.

2. Cottenceau, G.; Besson, T.; Gautier, V.; Rees, C.W.; Pons, A.M. Antibacterial evaluation of novel $\mathrm{N}$-arylimino-1,2,3-dithiazoles and $\mathrm{N}$-arylcyanothioformamides. Bioorg. Med. Chem. Lett. 1996, 6, 529-532.

3. Thiery, V.; Rees, C.W.; Besson, T.; Cottenceau, G.; Pons, A.M. Antimicrobial activity of novel $N$-quinolinyl and $N$-naphthylimino-1,2,3-dithiazoles. Eur. J. Med. Chem. 1998, 33, 149-153.

4. Joseph, R.W.; Antes, D.L.; Osei-Gyimah, P. Antimicrobial Compounds with Quick Speed of Kill. US Patent 5688744, 1997. 
5. Moore, J.E. Certain 4-Halo-5-aryl-1,2,3-dithiazole Compounds and their Preparation. US Patent $4059590,1977$.

6. Appel, R.; Janssen, H.; Haller, I.; Plempel, M. 1,2,3-Dithiazolderivate, Verfahren zu ihrer Herstellung Sowie ihre Verwendung als Arzneimittel. DE Patent 2848221, 1980.

7. Besson, T.; Rees, C.W.; Cottenceau, G.; Pons, A.M. Antimicrobial evaluation of 3,1-benzoxazin4-ones, 3,1-benzothiazin-4-ones, 4-alkoxyquinazolin-2-carbonitriles and $N$-arylimino-1,2,3dithiazoles. Bioorg. Med. Chem. Lett. 1996, 6, 2343-2348.

8. Mayer, R.; Foerster, E.; Matauschek, B. Verfahren zur Herstellung von Aromatisch oder Heteroaromatisch Substituierten Cyanthioformamiden. DD Patent 212387, 1984.

9. Konstantinova, L.S.; Rakitin, O.A. Synthesis and properties of 1,2,3-dithiazoles, Russ. Chem. Rev. 2008, 77, 521-546.

10. Rees, C.W. Polysulfur-nitrogen heterocyclic chemistry. J. Heterocycl. Chem. 1992, 29, 639-651.

11. Besson, T.; Dozias, M.J.; Guillard, J.; Rees, C.W. New route to 2-Cyano-benzothiazoles via N-Arylimino-1,2,3-dithiazoles. J. Chem. Soc., Perkin Trans. 1 1998, 3925-3926.

12. Rakitin, O.A.; Rees, C.W.; Vlasova, O.G. Direct synthesis of 2-Cyano-benzimidazoles and the generation of $\mathrm{S}_{2}$. Tetrahedron Lett. 1996, 37, 4589-4592.

13. Christoforou, I.C.; Koutentis, P.A.; Michaelidou, S.S. 1,2,3-Dithiazole chemistry in heterocyclic synthesis. ARKIVOC 2006, 7, 207-223.

14. Besson, T.; Guillaumet, G.; Lamazzi, C.; Rees, C.W. Synthesis of 3,1-benzoxazines, 3,1-benzothiazines and 3,1-benzoxazepines via $N$-arylimino-1,2,3-dithiazoles. Synlett 1997, 704-706.

15. Lee, H.; Kim, K. A new procedure to $\mathrm{N}$-arylcyanothioformamides from 5-arylimino-4-chloro-5H1,2,3-dithiazoles. Bull. Korean Chem. Soc. 1992, 13, 107-108.

16. Michaelidou, S.S.; Koutentis, P.A. The synthesis of 2-cyano cyanothioformanilides from 2-(4-chloro-5H-1,2,3-dithiazol-5-ylideneamino)benzonitriles using DBU. Synthesis 2009, 4167-4174.

17. Besson, T.; Emayan, K.; Rees, C.W. 1,2,3-Dithiazoles and new routes to 3,1-benzoxazin-4-ones, 3,1-benzothiazin-4-ones and $N$-arylcyanothioformamides. J. Chem. Soc. Perkin Trans. 1 1995, 2097-2102.

18. Lee, H.; Kim, K. Reactions of 5-(arylimino)-4-chloro-5H-1,2,3-dithiazoles with primary and secondary alkylamines: Novel synthesis of (arylimino)cyanomethyl alkylamino disulfides and their mechanisms of formation. J. Org. Chem. 1993, 58, 7001-7008.

19. Besson, T.; Guillard, J.; Rees, C.W. Rapid synthesis of 2-cyanobenzothiazole, isothiocyanates and cyanoformanilide derivatives of dapsone. J. Chem. Soc. Perkin Trans. 1 2000, 563-566.

20. Besson, T.; Guillard, J.; Rees, C.W.; Thiéry, V. New syntheses of aryl isothiocyanates. J. Chem. Soc. Perkin Trans. 1 1998, 889-892.

21. Baraldi, P.G.; Pavani, M.G.; Nuñez, M.C.; Brigidi, P.; Vitali, B.; Gambari, R.; Romagnoli, R. Antimicrobial and antitumor activity of $n$-heteroimmine-1,2,3-dithiazoles and their transformation in triazolo-, imidazo-, and pyrazolopirimidines. Bioorg. Med. Chem. 2002, 10, 449-456.

22. Lee, H.-S.; Chang, Y.-G.; Kim, K. A facile synthesis of 3-substituted 2-cyanoquinazolin-4(3H)-ones and 3-alkyl-2-cyanothieno[3,2-d]pyrimidin-4(3H)-ones via 1,2,3-dithiazoles. J. Heterocycl. Chem. 1998, 35, 659-668. 
23. Cuadro, A.M.; Alvarez-Builla, J. 4,5-Dichloro-1,2,3-dithiazolium chloride (Appel's Salt): reactions with $N$-nucleophiles. Tetrahedron 1994, 50, 10037-10046.

24. English, R.F.; Rakitin, O.A.; Rees, C.W.; Vlasova, O.G. Conversion of imino-1,2,3-dithiazoles into 2-cyanobenzothiazoles, cyanoimidoyl chlorides and diatomic sulfur. J. Chem. Soc. Perkin Trans. 1 1997, 201-205.

25. Konstandinova, L.S.; Rakitin, O.A.; Rees, C.W.; Sivadasan, S.; Torrobas, T. New route to 2-cyanobenzimidazoles. Tetrahedron 1998, 54, 9639-9650.

26. Kalogirou, A.S.; Koutentis, P.A. The degradation of 4,5-dichloro-1,2,3-dithiazolium chloride in wet solvents. Tetrahedron 2009, 65, 6859-6862.

27. Bordwell pKa Table (Acidity in DMSO). Available online: http://www.chem.wisc.edu/areas/ reich/pkatable/ (accessed on 1 June 2011).

28. Stewart, R.; Harris, M.G. Comparison of the acidities and basicities of amino-substituted nitrogen heterocycles. J. Org. Chem. 1978, 43, 3123-3126.

29. L'abbe, G.; D'hooge, B.; Dehaen, W. Unusual behavior of 4,5-dichloro-1,2,3-dithiazolium chloride (Appel's salt) with 5-aminopyrazoles: A synthetic method of $1 H$-pyrazolo[3,4- $d]$-thiazoles. J. Chem. Soc., Perkin Trans. 1 1995, 2379-2380.

30. L'abbe, G.; Bastin, L.; Dehaen, W.; Toppet, S.; Delbeke, P.; Vlieghe, D.; van Meerveek, L. Reactions of 5-chloro-1,2,3-dithiazolium salts with activated methylene compounds. J. Chem. Soc., Perkin Trans. 1 1994, 2545-2551.

Sample Availability: Samples of the compounds are available from the authors.

(C) 2011 by the authors; licensee MDPI, Basel, Switzerland. This article is an open access article distributed under the terms and conditions of the Creative Commons Attribution license (http://creativecommons.org/licenses/by/3.0/). 